\title{
Gesichtspankte für die Gliederung der Sprachanalyse in Untersuchungsgebiete und die dominierende Rolle des Handlungscharakters der Sprache
}

1. Aus der dialektisch-materialistischen Philosophie lassen sich u. a. die folgenden Prinzipien ableiten, die wichtige Voraussetzungen für die Klärung methodologischer Fragen der Sprachwissenschaft darstellen: das Prinzip der theoretischen Gliederbarkeit komplexer Objekte, das Prinzip des Abbildcharakters der Erkenntnis, das Prinzip des relativen Charakters der Erkenntnis und das Prinzip der Steuerung des Erkenntnisprozesses durch gesellschaftliche Interessen.

2. Ein komplexes Objekt kann theoretisch nach verschiedenen Gesichtspunkten in Untersuchungsgebiete gegliedert werden. ${ }^{1} \mathrm{Im}$ Hinblick auf die Sprache kennen wir Gliederungen, die ihren Niederschlag in solchen Teildisziplinen der Sprachwissenschaft wie Grammatik, Lexikologie, Semantik, Stilistik, historische Spracb wissenschaft, Textlinguistik, Typologie u. a. m. finden. Andere Arten der Gliederung führen zu Grenzwissenschaften wie Psycholinguistik, Soziolinguistik, Ethnolinguistik. Sprachliche Fakten können auch in anderen Wissenschaften eine Rolle spielen. Dies ist z. B. der Fall in der kognitiven Psychologie, der Logik und der Literaturwissenschaft.

Eine Untersuchung der Grundlagen für die Gliederung der Sprachuntersuchung in verschiedene Gebiete, insbesondere für das Verhältnis dieser Gebiete zueinander in sachlicher und methodologischer Hinsicht, bietet eine Möglichkeit, die Dialektik des tatsächlichen Erkenntnisprozesses bezogen auf Sprache zu studieren und methodologische Postulate für dialektisch-materialistische Standpunkte in der Sprachanalyse zu entwickeln. Einige Vorüberlegungen zu einer solchen Untersuchung sollen hier zur Diskussion gestellt werden.

3. Eine Gliederung schafft Teilgebiete der Sprachanalyse, die durch Faktenmengen und ihre theoretischen Deutungen bestimmt sind. Die theoretische Deutung darf nicht heterogen sein, sie legitimiert die Betrachtung der Fakten als zusammenhängende Fakten eines Gebiets. In der Sprachwissenschaft werden z. B. Gebiete wie Phonologie, Morphologie, Syntax, Lexik unterschieden. Diese könnten wiederum als ein Gebiet, nämlich das der Grammatik, zusammengefa ßt und von anderen Gebieten abgegrenzt werden. Die Grundlage für eine solche Gliederung bilden offensichtlich systematisch zusammenhängende Faktenmengen und dazugehörige Systeme theoretischer Ordnung und Erklärung.

4. Für die Charakterisierung des Verhältnisses zwischen Objekt, Fakten und Theorie ist die Heranziehung der dialektisch-materialistischen Abbildtheorie wesentlich. Danach ist die Möglichkeit, zusammenhängende Faktenmengen herauszugliedern, nicht

1 Zur theoretischen Zerlegung eines Objekts in Forschungsgegenstände vgl. auch A. A. LEONT'Ev, Sprache, Sprechen, Sprechtätigkeit, übersetzt und herausgegeben von C. Hefschen und W. Stölting, Stuttgart, Berlin, Köln, Mainz 1971, S. $15 \mathrm{ff}$. 
willkürlich, sondern sie hängt von Eigenschaften des Objekts ab. Objektive Eigenschaften werden durch Faktenaussagen erfaBt, die der theoretischen Analyse vorgegeben sind. Was in der Sprachwissenschaft Fakten sind, d. h. Aussagen über als objektiv angenommene Eigenschaften einer Sprache, und wie das Verhältnis zwischen Fakten und Theorie zu bestimmen ist, so daß das Primat der Fakten vor der Theorie gesichert ist, ist eine noch keineswegs geklärte methodologische Frage der Sprachwissenschaft. 2

5. Eine Faktenmenge kann nicht als gegebene, endliche Menge von Aussagen aufgefaßt werden. Der Etablierung eines Untersuchungsgebiets entspricht eher die Annahme bestimmter ausgewählter Eigenschaften und Zusammenhänge, die gegeben sein müssen, damit Faktenaussagen als eine Menge erfaßt werden können. Damit ist aber die Bestinumung, ob eine Erscheinung zur Faktenmenge eines Gebiets gehört oder nicht, von bestimmten theoretischen Annahmen abhängig. So betrachtet ist die Festlegung von Faktenmengen nicht theorieunabhängig. Ein scharfer Gebietsbegriff würde von eindeutig bestimmten Theorien ausgehen müssen. Es scheint aber aus zwei Gründen nicht zweckmäßig zu sein, den Begriff in dieser Weise festzulegen: 1. Sind Theorien und die für die Konstituierung der Faktenmenge verantwortlichen Eigenschaften und Zusammenhänge meist nicht scharf ausformuliert, so daß unscharfe Faktenmengen anzunehmen sind. 2. Scheint es durchaus sinnvoll zu sein, zuzulassen, daB ein Gebiet durch wenigstens teilweise verschiedene Theorien bestimmt sein kann. Was wiederum Konsequenzen für die Faktenmenge hat.

6. Da eine Gebietsbestimmung immer nur aus der Sicht einer Theorie genau festgelegt werden kann, die theoretischen Annahmen aber sowohl in einer gegebenen Phase der Wissenschaft verschieden sein, als auch im Verlauf des Erkenntnisprozesses verändert werden können, darf keine vorgeschlagene Gliederung verabsolutiert werden. Jede Gliederung basiert immer nur auf relativ gesicherten Erkenntnissen. Durch methodologische Postulate muß erreicht werden, daß sowohl die Faktenmenge als auch die auf sie bezogene theoretische Deutung als relativ betrachtet werden. Zu berücksichtigen ist, da $B$ Veränderungen der theoretischen Basis entweder mehr oder weniger im Rahmen einer Faktenmenge bleiben oder aber zu einer Erweiterung der Faktenmenge führen können. Unterschiedliche strukturalistische Grammatiktheorien wie die IC-Grammatik, die Glossematik, die generative Grammatik blieben - bei allen z. T. gravierenden Unterschieden - ungeführ im Rahmen der gleichen Prinzipien für die zu untersuchende Faktenmenge. Eine erhebliche Erweiterung des Faktenbereichs trat erst ein, als semantische Fragestellungen in die Grammatiktheorie einbezogen wurden. In den letzten Jahren zeichnet sich die Notwendigkeit einer nochmaligen Erweiterung ab, und zwar durch die Einbeziehung von Fakten, die das Zusammenspiel von sprachlichen Åußerungen und kommunikativen Bedingungen sowie Funktionen betreffen. Die Rechtfertigung einer Gliederung muß deshalb die Zusammenhänge der ausgegliederten Faktenmenge mit anderen Fakten, die über das Objekt Sprache ermittelt werden können, berücksichtigen. Sie darf die Fakten, die mit einem Gebiet verbunden sind, nicht absolut sehen, d. h. nicht unabhängig von Tatsachen, die in anderen Gebieten betrachtet werden. Jedes durch eine Gliederung geschaffene Untersuchungsgebiet ist nur theoretisch absolut, nicht

2 Vgl. dazu W. Motsch, Grammatiktheorie und sprachliche Wirklichkeit. Zu einigen Fragen der empirischen Begründung linguistischer Aussagen, in: Linguistische Studien des ZISW, R. A, H. 40, Berlin 1977. 
sachlich. Dieser Unterschied wurde in strukturalistischen Schulen meist übersehen oder bewußt negiert. Eine Gliederung muß stets mit bestimmten Annahmen über die Beziehungen der Fakten eines Cntersuchungsgebiets zu Fakten anderer Gebiete verbunden sein, d. h. aber mit bestimmten Gesamtvorstellungen von Sprache. Eine solche Gesamtvorstellung liefert den Rechtfertigungs- und Orientierungshintergrund für die Gliederung insgesamt und für jedes einzelne Gebiet. Die mit der separaten Untersuchung der Fakten eines Gebiets einhergehende Abstraktion von anderen Gebieten muß methodologisch mit einer Reintegration der gewonnenen Erkenntnisse in Erkenntnisse und Fragestellungen über das gesamte Objekt verbunden werden.

7. Eine Gliederung wird nicht nur durch methodologische Postulate über das Verhältnis zwischen Objekt, Tatsachenaussagen und theoretischen Aussagen bestimmt, sondern auch durch das gesellschaftliche Interesse an Erkenntnissen über Sprache. Dies ist eine Konsequenz aus den Zusammenhang zwischen Erkenntnistätigkeit und gesellschaftlicher Praxis. Gesellschaftliches Interesse kann verschieden motiviert sein und auch in unterschiedlich deutlicher Form in Erscheinung treten. Ohne die komplizierte Problematik hier behandeln zu wollen, scheinen bezüglich Sprache zwei Aspekte eine wichtige Rolle zu spielen:

a) Das Erkenntnisinteresse wird durch die Möglichkeit, bestimmte Erkenntnisse über Sprache in größere ideologische Orientierungsräume einzuordnen, gelenkt, die ihrerseits Ausdruck der sozialen Interessen von Klassen sind. Beispiele sind: die Orientierung der deutschen Sprachwissenschaft des 19. Jahrhunderts an der NationProblematik; die Lenkung sprachwissenschaftlicher Forschungsthemen und Theorien durch den positivistischen Scientismus der 40er und 50er Jahre; Bemühungen um eine Bereicherung des Marxismus-Leninismus durch sprachtheoretische Erkenntnisse.

b) Das Erkenntnisinteresse wird durch Möglichkeiten gelenkt, Forschungsergebnisse praktisch auszuwerten. Beispiele sind: Bemühungen um sprachwissenschaftliche Grundlagen für wirkungsvollere Lehrmethoden und -mittel auf dem Gebiet des Sprachunterrichts; Forschungen zur automatischen Verarbeitung natürlicher Sprache.

Welche Gebiete durch eine Gliederung etabliert werden können, ist von den wirklichen Eigenschaften von Sprache, sowie vom Stand unserer Kenntnisse abhängig. Die Bevorzugung oder Vernachlässigung schon etablierter Gebiete einerseits und die Auffindung neuer Gebiete andererseits wird dagegen ma Bgeblich durch gesellschaftliche Interessen gelenkt.

8. Einige Fragen, die sich aus der Anwendung der bisher entwickelten Gedanken auf aktuelle methodologische und theoretische Fragen der Sprachwissenschaft ergeben, sollen kurz am Beispiel der Generativen Grammatik und der Sprechaktanalyse diskutiert werden.

In den methodologischen Voraussetzungen der generativen Grammatik ist das Verhältnis zwischen Objekt, Fakten und Theorie nicht geklärt. Gegenstand einer Grammatiktheorie ist nach CHoMsKY die grammatische „Kompetenz" eines „idealen“" Sprecher/Hörers.4 Es bleibt aber unbestimmt, wie die Fakten des Gebiets gramma-

3 Vgl. W. Necmann, Über Probleme und Prozesse bei der Bestimmung des Gegenstancies der Linguistik, in: Linguistische Studien des ZISW, R. A, H. 40, Berlin 1977, S. $22 \mathrm{ff}$.

4 Vgl. N. Cномsкy, Aspekte der Syntax-Theorie. Deutsche Übersetzung, Berlin 1970, S. $13 \mathrm{ff}$. 
tischer Untersuchungen, die ja nur von der Kompetenz wirklicher Sprecher/Hörer ausgehen könmen, als Aussagen ïher die Kompetenz eines ,idealen“ Sprecher/Hörers zu gewinnen sind.

In Chonskrs Andeutungen zu dieser Problematik findet man nur vage Hinweise. So soll z. B. abgesehen werden von:

- psychologischen und physiologischen Zufälligkeiten der Rede;

- psycho-physiologischen Voraussetzungen und Prozessen des Sprechens (GedankenÄußerns) und Hörens (Gedanken-Verstehens);

- sozialen Besonderheiten wie unterschiedliche Sprachbeherrschung, unterschiedliche Soziolekte u. a.

Wesentliche Probleme werden von Chomsky nicht diskutiert:

a) Aus der sozialen Position eines Menschen ergeben sich nicht nur Unterschiede im Grad der aktiven Beherrschung einer Sprachausprägung (z. B. Hochsprache), sondern vor allem auch Unterschiede hinsichtlich der Kenntnis und Bewertung der differierenden Existenzformen und Stilschichten einer Nationalsprache. Insbesondere spielen die Auswirkungen unterschiedlicher Weltkenntnisse und ideologischer Wertungen und Einstellungen auf semantische Systeme eine Rolle. Probleme und Fakten dieser Art können durch einen idealen Sprecher/Hörer nicht erfaßt werden. Sie aus der Untersuchung auszuschließen ist sachlich nicht zu motivieren. CHomskYs Idealisierungsbasis ist deshalb genau an den Punkten willkürlich, wo sozial spezifische Fakten a priori aus der Untersuchung grammatischer Fakten ausgeschlossen werden. Die Konsequenz ist eine Einschränkung der Fakten auf Aussagen über grammatische Eigenschaften homogener Sprachformen (Hochsprache, einzelne Dialekte usw.). Zusammenhänge zwischen den Existenzformen und stilistisch-funktionalen Ausprägungen einer Sprache, die neben sozial-kommunikativen auch „rein“ grammatische Aspekte enthalten, gehören nach CHomsky nicht zu den Fakten, die die Grammatiktheorie untersucht.

b) Сномsкy setzt bei seiner Interpretation der Saussureschen Trennung von langue und parole ein „rein" psychologisches Handlungskonzept voraus. 5 "Performance“" ist für ihn ein Gebiet, in dem psychologische Prozeduren des Sprechens oder Hörens untersucht werden, soweit sie mit der Anwendung der „linguistic competence“ verbunden sind. "Linguistic competence“ umfaßt aber keine sozial spezifischen Fakten und beruht implizit auf einem Sprachkonzept, das Sprache nur als Mittel zum Ausdruck von Gedanken über die Welt versteht und nicht als ein Instrument gesellschaftlicher Tätigkeit, als ein Instrument, das spezifische Funktionen im gesamten Lebensprozeß der Menschen einer Gesellschaft erfüllt. Daß sich aus den Funktionen von sprachlichen Mitteln Fakten und theoretische Probleme für das Gebiet grammatischer Untersuchungen ergeben können, bleibt bei Сномsкx unberiicksichtigt. Tatsächlich läßt sich leicht zeigen, daß Bedingungen und Zielstellungen sprachlichkommunikativen Handelns (wie Einstellungen und Wertungen des Sprechers oder Hörers, soziale Beziehungen zwischen ihnen, Absicht einer Sprechhandlung, die der Hörer erkennen soll, damit die Handlung glïckt) nicht aus der Untersuchung gram-

5 Vgl. dazu W. Motsch, Sprache als Handlungsinstrument, in: Linguistische Studien des ZISW, R. A, H. 19, Berlin 1975, S. $11 \mathrm{ff}$. 
matischer Mittel ausschließen läßt, weil entsprechende Tatsachen in die LautBedeutungsbeziehungen eingehen.

Es zeigt sich, daB die grundsätzliche Ausklammerung von sogenannten pragmatischen Faktoren auf willkürlichen Voraussetzungen ïber das Gebiet der Grammatik beruhen. Letztlich auf einer sehr einseitigen Gesamtrorstellung von Sprachen, die die Funktion von Aussagen überbewertet. Aussagen lassen sich am ehesten als sprachliche Formen untersuchen, deren Struktur allein durch Beziehungen zwischen Lautgebilden und semantischen Konstrukten charakterisiert ist.

Die Einbeziehung bestimmter „pragmatischer" Fragen in die Grammatik ist nicht eine einfache Erweiterung des Untersuchungsgebiets der Grammatik, sondern die Korrektur einer Gliederung, die auf ein dem Objekt nicht angemessenes Sprachkonzept zurückgeht, auf ein Konzept, das alle Eigenschaften natürlicher Sprache als uninteressant ausschließt, die Sprachen zu Instrumenten gesellschaftlicher Tätigkeit befähigen. ChomsKY sucht denn auch ganz explizit das Wesen natürlicher Sprachen (the nature of language) in der Deutung von sozial und historisch indifferenten Eigenschaften.

Wenn man dagegen in Betracht zieht, da B in der Grammatik einer Sprache Mittel angelegt sind, deren Funktion darin besteht, eine Sprache als Handlungsinstrument zu befähigen, ergibt sich eine entscheidende methodologische Konsequenz: das Verhältnis zwischen langue - parole, competence - performance, Sprachsystem - Sprachgebrauch ist nur durch die Voraussetzung dialektischer Beziehungen zu erfassen. Eine strikte Abgrenzung, deren empirische Rechtfertigung nicht systematisch betrachtet wird, führt zu willkürlichen Beschränkungen der Faktenbasis der Grammatik. Der Handlungsaspekt bildet nicht nur die Grundlage für die Etablierung neuer, relativ separater Untersuchungsgebiete der Sprachwissenschaft, sondern er hat eine dominierende Funktion bei der Gliederung der Sprachanalyse in Untersuchungsgebiete. Er stellt wesentliche Züge des Gesamtkonzepts von Sprache dar und hat orientierende Funktion für die Darstellung der Zusammenhänge zwischen den in unterschiedlichen Gebieten untersuchten Faktenmengen.

In der - wie gezeigt wurde - willkürlichen Beschränkung der Sprachanalyse auf sozial indifferente Eigenschaften natürlicher Sprachen kommt ein ideologischer Interessenraum zum Ausdruck, der eine Spielart der bürgerlichen Weltanschauung in unserer Zeit ist.7

9. Die Berücksichtigung des Handlungsaspekts als dominierenden Hintergrund für die Bestimmung des Untersuchungsfeldes der Grammatiktheorie und ihrer Einordnung in ein Gesamtkonzept von Sprache ist nicht identisch mit dialektisch-materialistischen Positionen. Gegenwärtig gibt es auch in der nicht-marxistischen Sprachwissenschaft zahlreiche Versuche, die Einengung der Sprachanalyse und ganz speziell auch der Grammatikforschung durch eine handlungstheoretische Fundierung zu überwinden. In diesem Zusammenhang ist vor allem die auf John L. Austn zurückgehende Sprechaktanalyse zu erwähnen. Insbesondere das von John R. SEarLe in

6 Eine Zusammenstellung grammatischer Fragestellungen, die mit. Faktoren der Kommunikstion verbunden sind, wurde bereits 1970 von $D$. WoNDERLICH vorgenommen (D. Wondfrlich, Die Rolle der Pragmatik in der Linguistik, in: Der Deutschunterricht $22(1970)$, S. 5-41).

7 Vgl. dazu W. Motsch, Zur Kritik des sprachwissenschaftlichen Strukturalismus, Berlin 1974 , S. $160 \mathrm{ff}$. 
.enger Anlehnung an Adstiv entwickelte Paradigma für die Untersuchung von Sprechakten weist wesentliche Mängel auf, die hier nur pauschal angedeutet werden können: 1. Das volle Spektrum von Fragestellungen, die mit sprachlichem Handeln verbunden sind, wird von vornherein stark eingeschränkt, weil nur Einzelhandlungen unter dem Aspekt von Funktionen wie 'Frage', 'Aufforderung', 'Entschuldigung', 'Versprechen' usw. betrachtet werden, nicht die Rolle solcher Handlungen in komplexen Handlungsabläufen.

2. Man geht nicht von wirklichen Handlungen aus, von einer Analyse konkreter sozialer Situationen, in denen Fragen gestellt, Versprechen abgegeben, Aufforderungen erteilt werden, sondern von Selbstbefragungen. Wenn dieses Verfahren methodologisch nicht abgesichert wird, können sich schwerwiegende Konsequenzen für die Zuverlässigkeit wissenschaftlicher Aussagen ergeben.

3. Es existiert kein orientierendes Konzept von gesellschaftlichem Handeln, das es ermöglicht, Sprechhandlungen durch geeignete Arbeitshypothesen auf dem Hintergrund gesellschaftlicher Tätigkeit zu studieren. ${ }^{8}$

4. Es wird wiederum ein ,idealer" Sprecher/Hörer angenommen, der alle Sprechakttypen (illocutionary forces) seiner Sprache beherrscht. Die soziale Spezifik und Differenz in einer konkreten Gesellschaft sowie in der historischen Entwicklung wird nicht thematisiert. Als Konsequenz ergibt sich die HaBermas'sche „Universalpragmatik“, die nach universellen gesellschaftlichen Voraussetzungen kommunikativen Handelns sucht. 9 Der gesellschaftliche Charakter der Sprache wird auf diese Weise zwar nicht ausgeschlossen, jedoch auch nicht in seinem historischen Wesen verstanden, speziell in seiner durch den aktuellen Stand des Klassenkampfes in verschiedenen Gesellschaften determinierten Spezifik.

Auf dem Gebiet der Sprachtheorie im Sinne von Austin und Searle wird, trotz wichtiger Fortschritte gegenüber CHoMsKYs Orientierung der Sprachwissenschaft, der alte Isolationismus als Denkhaltung fortgesetzt.

8 Vgl. dazu die Kritik von K. EmuCH, Thesen zur Sprechaktheorie, in: D. WUNDFRLTOH (Hrsg.), Linguistische Pragmatik, Frankfurt/M. 1972, S. 122-126, sowie im gleichen Band das Alternativmodell zum Studium von Sprechakten K. EHIICH, J. REHBEIN, Zur Konstitution pragmatischer Einheiten in einer Institution: Das Speiserestaurant, S. 209-254.

$9 \mathrm{Vgl}$. J. Haberuss, Was heißt Universalpragmatik?, in: K. O. APEL (Hrsg.), Sprachpragmatik und Philosophie, Frankfurt/M. 1976, S. 174-272. 